\section{Preventing cardiac damage in patients with COVID-19}

Researchers have identified a pathway associated with cardiac injury in patients with coronavirus disease 2019 (COVID-19). Inhibitors of this pathway prevented and rescued cardiac dysfunction in human cardiac organoids (hCO) and a mouse cytokine-storm model.

"It is becoming increasingly evident that patients with severe acute respiratory syndrome coronavirus 2 (SARS-CoV-2) infection can develop cardiac complications, and those with pre-existing cardiovascular disease are at greater risk," comments first author Richard Mills. "We sought to understand how SARS-CoV-2 could be driving cardiac dysfunction through possible direct (cardiac infection) and indirect (inflammation or cytokine storm) means, observed in many patients," he continues.

The effects on cardiac function of a range of pro-inflammatory cytokines that have been shown to be elevated in patients with COVID-19 were screened in a pluripotent stem cell-derived hCO model. A combination of IFN- $\gamma$, IL- $1 \beta$ and poly(I:C), which the researchers termed 'cardiac cytokine storm', induced the most consistent, robust diastolic dysfunction in the hCO model. Treatment with the cytokine storm cocktail induced increases in 91 phosphosites, including phosphorylation of signal transducer and activator of transcription 1 (STAT1) and two sites on bromodomain-containing protein 4 (BRD4). Furthermore, single-nuclei RNA sequencing of the hCOs revealed that the cytokine storm treatment induced a robust viral-like response in multiple cell populations that was predicted to be mediated via STAT1 and BRD4.

Strikingly, delayed injection of a bromodomain and extraterminal family (BET) inhibitor $1.5 \mathrm{~h}$ after lipopolysaccharide (LPS) injection fully prevented the decrease in cardiac function in an LPS-induced cytokine-storm mouse model.

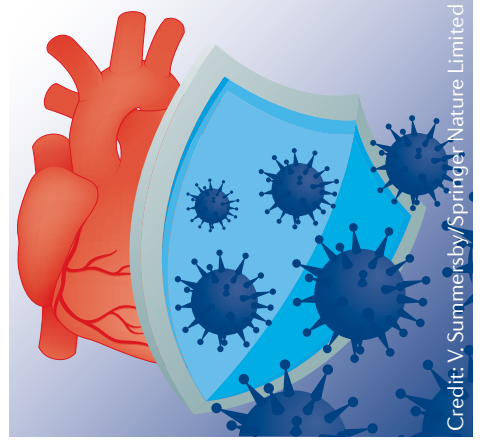

Furthermore, in a mouse model of SARS-CoV-2 infection, treatment with a BET inhibitor decreased the transcription of genes related to the viral response. "Using a number of omic-based approaches, we identified a therapeutically targetable mechanism, BRD4 activation along with a STAT1-mediated viral response, in which BET inhibition could prevent and recover cardiac dysfunction," states Mills.

Finally, treatment with the commercially available bromodomain 2 (BD2)-selective BET inhibitor apabetalone reduced the cytokine storm-induced diastolic dysfunction and reduced angiotensin converting enzyme 2 levels and viral infection in cardiomyocytes. These data suggest that $\mathrm{BD} 2$-selective $\mathrm{BET}$ inhibitors are leading candidates for rapid clinical translation for the prevention of cardiac injury in patients with COVID-19.

"We believe that BET inhibitors are attractive candidates for many indications in which inflammation could drive cardiac dysfunction, including other viral infections, sepsis and myocarditis," remarks Mills. "Owing to the lack of effective treatments for many of these diseases, BET inhibitors potentially have huge implications for patient care," he concludes.

Andrew Robson

ORIGINAL ARTICLE Mills, R. J. et al. BET inhibition blocks inflammation-induced cardiac dysfunction and SARS-CoV-2 infection. Cell https://doi.org/10.1016/j.cell.2021.03.026 (2021) RELATED ARTICLE Nishiga, M. et al. COVID-19 and cardiovascular disease: from basic mechanisms to clinical perspectives. Nat. Rev. Cardiol. 17, 543-558 (2020)

\section{IN BRIEF}

\section{COVID - 19}

\section{Congenital heart disease and risk of severe COVID}

Adult patients with congenital heart disease are at increased risk of a complicated disease course with coronavirus disease 2019 (COVID-19). In a study conducted in 25 European centres, the EPOCH investigators identified 105 adults with congenital heart disease who had been diagnosed with COVID-19. Of these patients, 13 had a complicated disease course, defined as hospitalization requiring non-invasive or invasive ventilation and/or inotropic support or death. Risk factors for a complicated disease course included age (OR 1.3), multiple comorbidities (OR 7.1), obesity (OR 7.2) and cyanotic heart disease (OR 13.2), including unrepaired cyanotic defects or severe pulmonary hypertension with Eisenmenger syndrome. ORIGINAL ARTICLE Schwerzmann, M. et al. Clinical outcome of COVID-19 in patients with adult congenital heart disease. Heart https://doi.org/10.1136/heartjnl-2020-318467 (2021)

\section{HEART FAILURE}

\section{Al identifies distinct subgroups of HFpEF}

An artificial intelligence (Al) analysis has identified four subgroups of patients with heart failure with preserved ejection fraction (HFpEF), with distinct biomarker profiles, clinical characteristics and outcomes. A machine learning unsupervised cluster analysis was performed using 363 biomarkers from 429 patients with HFpEF. Four distinct clusters of patients were identified: cluster 1 with the highest prevalence of diabetes mellitus and renal disease; cluster 2 with the oldest age and frequent age-related comorbidities; cluster 3 with the youngest age, largest body size and least severe symptoms; and cluster 4 with the highest prevalence of ischaemic disease, smoking and chronic lung disease and the most severe symptoms. Death and hospitalization for heart failure were highest in clusters 1 and 4 and lowest in cluster 3 , indicating that subgroups of patients with $\mathrm{HFpEF}$ require specific therapies tailored to their particular clinical phenotype and underlying pathophysiological mechanisms.

ORIGINAL ARTICLE Woolley, R. J. et al. Machine learning based on biomarker profiles identifies distinct subgroups of heart failure with preserved ejection fraction. Eur.J. Heart Fail. https://doi.org/10.1002/ejhf.2144 (2021)

\section{CARDIAC DEVELOPMENT}

\section{Progesterone receptor controls heart maturation}

Progesterone signalling via the progesterone receptor has an important role in controlling post-natal maturation of both male and female human hearts. Researchers performed single-nucleus RNA-sequencing of 54,140 nuclei from diverse cardiac cell types from nine human donors to investigate transcriptional changes during maturation from fetal stages to adulthood. Bulk RNA-sequencing and ATAC-sequencing were then performed on purified cardiomyocyte nuclei from 21 human donors to validate the transcriptional changes and to investigate chromatin accessibility. The progesterone receptor was identified as an important mediator of sex-dependent transcriptional programmes associated with oxidative metabolism and maturation of cardiomyocyte contractile properties. Although serum progesterone levels increase 20 -fold during the luteal phase of the reproductive cycle in women, progesterone levels are similar in men and women outside of the luteal phase, suggesting that progesterone might regulate cardiac physiology in both sexes.

ORIGINAL ARTICLE Sim, C. B. et al. Sex-specific control of human heart maturation by the progesterone receptor. Circulation https://doi.org/10.1161/CIRCULATIONAHA.120.051921 (2021) 